\title{
Long-lived memory for electronic spin in a quantum dot: Numerical analysis
}

\author{
V. V. Dobrovitski, ${ }^{1}$ J. M. Taylor, ${ }^{2}$ and M. D. Lukin ${ }^{2}$ \\ ${ }^{1}$ Ames Laboratory, Iowa State University, Ames, IA 50011, USA \\ ${ }^{2}$ Department of Physics, Harvard University, Cambridge, Massachusetts 02138, USA
}

\begin{abstract}
Techniques for coherent control of electron spin-nuclear spin interactions in quantum dots can be directly applied in spintronics and in quantum information processing. In this work we study numerically the interaction of electron and nuclear spins in the context of storing the spin-state of an electron in a collective state of nuclear spins. We take into account the errors inherent in a realistic system: the incomplete polarization of the bath of nuclear spins and the different hyperfine interactions between the electron and individual nuclei in the quantum dot. Although these imperfections deteriorate the fidelity of the quantum information retrieval, we find reasonable fidelities are achievable for modest bath polarizations.
\end{abstract}

PACS numbers: 03.67.-a,75.40.Mg,73.21.La,76.70.-r

\section{INTRODUCTION}

Currently, the development of techniques for coherent manipulation and control of electron spins in semiconductor nanostructures is an area of vibrant research activity ${ }^{1,2,3,4,5,6}$. Such techniques are needed for many coherent spintronic devices ${ }^{7}$, such as a Datta-Das transistor ${ }^{8}$, and for quantum computing using the spin of an electron in a quantum dot (QD) as a qubit 1.5 . However, the decoherence time of an electron spin constitutes a fundamental obstacle on the way to realization of a QDbased quantum computation 9,10 . In order to enhance the coherence time, a quantum memory protocol has been recently suggested ${ }^{11}$ which allows mapping of the quantum state of the electron spin on the collective spin of the nuclei present in the QD, thereby taking advantage of the intrinsically longer coherence times of nuclear spins. Under ideal circumstances, the proposed scheme allows to encode and, at later time, to retrieve, the electron spin state with near $100 \%$ reliability. Furthermore, it can be extended to implementing arbitrary 1- and 2-qubit operations $^{12}$. Imperfections, which should be expected in realistic settings, lead to errors, thus deteriorating the fidelity of the quantum memory protocol11.13. Some errors can be reduced ${ }^{14.15}$, but some are inherent to the protocol, such as the incomplete polarization of the nuclear spins and the spread in the hyperfine interactions between the electron and the nuclei in the QD. In this work, we use numerical simulations ${ }^{16,17}$ to analyze the effect of these errors on the performance of the quantum memory protocol. We show that the scheme remains feasible for the spin bath polarizations exceeding $80 \%$, with the minimum fidelity of $\sim 80 \%$.

First, let us recall the scheme of Ref. 11. The electron with a spinor wave function $\left|\phi_{0}\right\rangle=\alpha|\downarrow\rangle+\beta|\uparrow\rangle$ is injected into an empty quantum dot, and starts interacting with the bath of $N$ nuclear spins in the dot. The most important part of this interaction is the isotropic contact hyperfine coupling. The total Hamiltonian of the system, taking into account the Zeeman energy of the electron spin in the external field $H_{0}$, becomes

$$
\mathcal{H}=g_{e}^{*} \mu_{B} H_{0} S^{z}+\sum_{k=1}^{N} A_{k} \mathbf{I}_{k} \mathbf{S}
$$

where $S=1 / 2$ is the electron spin, $I_{k}=3 / 2$ are the spins of the nuclei (the fact that the nuclear spins of $\mathrm{Ga}$ and As are not $1 / 2$ is not important here, up to renormalization of the appropriate parameters, and we will consider them as spin- $1 / 2$ in the following discussion), and $A_{k}=(8 \pi / 3) g_{e} \mu_{B} g_{n} \mu_{n}\left|\Psi\left(\mathbf{x}_{k}\right)\right|^{2}$ is the contact coupling determined by the electron density $\left|\Psi\left(\mathbf{x}_{k}\right)\right|^{2}$ at the site $\mathbf{x}_{k}$ of $k$-th bath spin, and by the Landé factors $g_{e}, g_{n}$ and the magnetons $\mu_{B}, \mu_{n}$ of the electron and the nuclei, respectively. At maximum polarization, the nuclei can produce an effective field $\sum_{k} A_{k} I_{k} / g_{e}^{*} \mu_{B}=5.2$ Tesla 18 .

Other terms in the Hamiltonian (11) can be omitted when discussing the storage and retrieval processes due to their smallness at the relevant timescales. These are, e.g., the Zeeman energy of the nuclear spins, the dipolar coupling between the bath spins, or between the electron spin and the bath spins. Also, we assume that the injection is performed quickly, so that the approximation of the sudden change of the Hamiltonian is applicable, and neither the state of the bath nor the state of the electron spin are affected by the injection process. Alternatively, fast control of the Zeeman splitting of the electron allows for the electron spin-nuclear spin system to be brought into resonance in a sudden approximation, and is similar in nature to the controlled injection/ejection of electrons from the quantum dot.

If the electronic wave function were spread uniformly over the dot volume, making all $A_{k}$ equal to $A$, and if the nuclear spins were completely polarized (i.e., if the bath were in the state $\left.\left|\chi_{0}\right\rangle=|\downarrow, \downarrow, \ldots \downarrow\rangle\right)$ then, for the external field adjusted to be $H_{0}=A(N-1) /\left(2 g_{e} \mu_{B}\right)$, the motion of the compound system (the electron and the bath spins) would become a sinusoidal oscillation between the states $\left|\phi_{0}\right\rangle \otimes\left|\chi_{0}\right\rangle$ and $|\downarrow\rangle \otimes\left[\alpha\left|\chi_{0}\right\rangle+i \beta\left|\chi_{1}\right\rangle\right]$ where $\left|\chi_{1}\right\rangle=(1 / \sqrt{N}) \sum_{k} S_{k}^{+}\left|\chi_{0}\right\rangle$ ( $N$ is the number of the nuclei in the dot). Thus, after half-period of such oscillations, the initial state of the electron spin becomes 
encoded in the state of the bath, and the electron can be ejected from the quantum dot. In order to retrieve this information after some time, another electron in the state $|\downarrow\rangle$ can be injected, and after a half-period of the oscillations, the state of the electron spin is again $\left|\phi_{0}\right\rangle=\alpha|\downarrow\rangle+\beta|\uparrow\rangle$. Of course, for this two-step scheme to work, the delay between the information encoding and retrieval should be small in comparison with the characteristic times of the omitted terms in the Hamiltonian (in practice, these terms should be reduced using, e.g., NMR techniques $\left.{ }^{19,20}\right)$.

However, real baths are incompletely polarized and the approximation of complete polarization is never applicable to a real bath with $N \sim 10^{4}-10^{6}$. To explain this, let us consider a highly polarized state of the bath described by a density matrix $\rho=(1 / Z) \exp \left(-\gamma I^{z}\right)$, where $I^{z}=\sum_{k} I_{k}^{z}$, and $Z$ is chosen such that $\operatorname{Tr} \rho=1$. For such a bath, the polarization is $P=\tanh \gamma / 2$, and for large $\gamma$ the value of $\Delta P=1-P$ is very small. However, the statistical weight $w(M)$ of the states with a given value $M$ of $I^{z}$ is $w(M)=C_{k}^{N} \theta^{k}(1-\theta)^{N-k}$, where $\theta=e^{\gamma} /\left(1+e^{\gamma}\right)$, the integer $k=N / 2-M$, and $C_{k}^{N}$ is a binomial coefficient. The distribution $w(M)$ is approximately Gaussian centered at $\bar{M}=(-N / 2)(1-\Delta P)$ with the $\operatorname{rms} \sigma^{2}=(N / 4) \Delta P(2-\Delta P)$. Therefore, unless $\Delta P$ is unrealistically small $\left(\Delta P \ll 1 / \sqrt{N} \sim 10^{-2}\right)$, a very large number of the bath states are involved in the time evolution, as opposed to only two states $\left|\chi_{0}\right\rangle$ and $\left|\chi_{1}\right\rangle$ involved in the case of $A_{k}=A$ and $\Delta P=0$. All these states have different oscillations frequencies, and form complex time-dependent superpositions, so that the fidelity of the information encoding and retrieval is reduced. Therefore, the errors associated with $\Delta P>0$ (incomplete polarization) and $A_{k} \neq A$ (spread in the hyperfine couplings) are intrinsic to the protocol. Curiously, the symmetry properties of the spin interaction allows for some correction, by state engineering and spin-echo techniques, for these errors 14 .

The paper is organized as follows. In section II, we consider qualitatively the analytical behavior of the storage protocol when inhomogeneity is negligible $\left(A_{k}=A\right)$ but polarization is less than perfect, and indicate how many of the effects of different oscillation frequencies and Hilbert space dimension lead to only moderate decreases in the fidelity of storage and retrieval. We then evaluate the same situation numerical in section III, and develop the useful measures of performance for the protocol. In section IV, the numerical results are extended to the case of inhomogeneous interaction $\left(A_{k} \neq A_{k^{\prime}}\right)$, and then in section $\mathrm{V}$ the minimal fidelity of the protocol (minimized over all possible electron spin states) is calculated.

\section{QUALITATIVE ANALYTICS FOR $A_{k}=A$ : WHY THE PROTOCOL WORKS FOR} $1 / \sqrt{N} \ll \Delta P \ll 1$

As indicated above ${ }^{11}$, for $A_{k}=A$ and $\Delta P>0$ the fidelity of the quantum memory protocol remains high if $\Delta P \ll 1$, even for $\Delta P \gg 1 / \sqrt{N}$. It is instructive to understand this robustness in detail.

To grasp the idea, let us consider only the first, encoding, step. We note that for $A_{k}=A$, the total spin of the nuclear bath $I$ is an integral of motion. If the initial state of the bath is $\left|I_{0}, M_{0}\right\rangle$, where $I_{0}$ is the value of $I$ and $M_{0}$ is the value of $I^{z}$, then the time-dependent wave function of the compound system is

$$
\begin{aligned}
|\psi(t)\rangle & =\beta\left|\psi_{1}(t)\right\rangle+\alpha\left|\psi_{2}(t)\right\rangle \\
\left|\psi_{1}(t)\right\rangle & =\left(\cos \omega_{1} t-i \xi_{1} \sin \omega_{1} t\right)|\uparrow\rangle \otimes\left|I_{0}, M_{0}\right\rangle \\
& -i \eta_{1} \sin \omega_{1} t|\downarrow\rangle \otimes\left|I_{0}, M_{0}+1\right\rangle \\
\left|\psi_{2}(t)\right\rangle & =\left(\cos \omega_{2} t+i \xi_{2} \sin \omega_{2} t\right)|\downarrow\rangle \otimes\left|I_{0}, M_{0}\right\rangle \\
& -i \eta_{2} \sin \omega_{2} t|\uparrow\rangle \otimes\left|I_{0}, M_{0}-1\right\rangle
\end{aligned}
$$

where $\omega_{1}=\sqrt{h_{1}^{2}+A^{2} C_{1}^{2} / 4}, \xi_{1}=h_{1} / \omega_{1}$, and $\eta_{1}=$ $A C_{1} /\left(2 \omega_{1}\right)\left(\omega_{2}, \xi_{2}\right.$ and $\eta_{2}$ are defined similarly). The relevant matrix elements are

$$
\begin{aligned}
h_{1} & =g_{e} \mu_{B} H_{0} / 2+A\left(M_{0}+1 / 2\right) / 2, \\
h_{2} & =g_{e} \mu_{B} H_{0} / 2+A\left(M_{0}-1 / 2\right) / 2, \\
C_{1} & =\sqrt{\left(I_{0}-M_{0}\right)\left(I_{0}+M_{0}+1\right)} \\
C_{2} & =\sqrt{\left(I_{0}+M_{0}\right)\left(I_{0}-M_{0}+1\right)} .
\end{aligned}
$$

The oscillation frequencies $\omega_{1,2}$ are strongly dependent on both $I_{0}$ and $M_{0}$. For the bath's initial density matrix $\rho=(1 / Z) \exp \left(-\gamma I^{z}\right)$, the statistical weight for the state $\left|I_{0}, M_{0}\right\rangle$ is

$$
w\left(I_{0}, M_{0}\right)=w\left(M_{0}\right)\left(C_{m}^{N}-C_{m-1}^{N}\right) / C_{k}^{N}
$$

where $k=N / 2-M_{0}$ and $m=N / 2-I_{0}$ are integers, and $C_{k}^{N}$ is a binomial coefficient. For $\Delta P \ll 1$, both $N-k$ and $m$ are small (note that $M_{0}<0$, so that $k$ is close to $N)$, and for a given $M_{0}$, it is easy to see that $w\left(I_{0}, M_{0}\right) \sim \Delta P^{(N-k-m)}$. The main contribution to the evolution comes only from the states with $I_{0}=-M_{0}$, and the states with larger values of $I_{0}\left(>-M_{0}\right)$ have too small a weight to impact the oscillations, and, correspondingly, to reduce the fidelity. Thus, the distribution of the frequencies $\omega_{1,2}$ in Eq. 2 is determined only by $w\left(M_{0}\right)$, and a spread of the oscillation frequencies $\sigma \sim \sqrt{N \Delta P}$ is much smaller than the mean frequency $\bar{\omega} \sim \sqrt{N}$.

Using Eqs. 23 it is easy to obtain an analytical approximation for $N \gg 1$ and $1 / \sqrt{N} \ll \Delta P \ll 1$ in the leading order in $\Delta P$ (but uniformly in time), since $\xi_{1}, \eta_{2} \sim 0$, $\xi_{2}, \eta_{1} \sim 1$. For the spin initially polarized along the $x$ axis $(\alpha=\beta=1 / \sqrt{2})$,

$$
s_{x}(t)=\cos \left(\frac{\tau}{2} \sqrt{N(1-\Delta P)}\right) \exp \left(-\frac{\sigma^{2} \tau^{2}}{8}\right)+O(\Delta P)
$$


while for the initial "spin-up" state,

$$
s_{z}(t)=\cos \left(\frac{\tau}{2} \sqrt{N(1-\Delta P)}\right)+O(\Delta P)
$$

where $\tau=A t$, and only $s_{x}(t)$ is influenced in the leading order in $\Delta P$. The difference between the results for transverse $(\mathrm{x})$ and parallel $(\mathrm{z})$ states indicates that the memory protocol is not isotropic: its performance depends in part upon the spin state being stored. As such, in analyzing errors, we will consider several different performance measures, described in detail below. We first consider the reliability of numerical simulations.

\section{QUANTITATIVE ANALYTICS FOR $A_{k}=A$ AND RELIABILITY OF COMPUTATIONS FOR SMALL $N$}

We focus on the case of $A_{k}=A$ in order to assess reliability of our simulations with $N=20$ (presented below, performed for the case of $A_{k} \neq A$ ), and to quantify the fidelity in detail, without omission of higher-order terms in $\Delta P$. The case of $A_{k}=A$ is a good check since it allows analytical solution, so that the results for $N=20$ can be directly compared with the results for realistic value $N \sim 10^{4}$.

Let us focus first on the encoding step to determine what will be useful performance measures for the protocol. Since $h_{1} \neq h_{2}$ in Eqs. 2] the components $S^{x}, S^{y}$ of the electron spin rotate with time in the $x-y$ plane with the angular velocity of order of $A / 4$. This rotation is uniform, and does not depend on the particular values $s_{x}, s_{y}$ of $S^{x}$ and $S^{y}$, because the Hamiltonian (11) is invariant with respect to the rotation in the $x-y$ plane $(\mathcal{H}$ commutes with $\exp \left[-i \epsilon\left(S^{z}+I^{z}\right)\right]$ for any $\left.\epsilon\right)$. As a result, even in perfect situation of $\Delta P=0, A_{k}=A$, for the initial value of $s_{y}^{(i)}=0$, the final retrieved value $s_{y}^{(f)} \neq 0$, and $s_{x}^{(f)} \leq s_{x}^{(i)}$. However, this error is noticeable only for relatively small $N(N \leq 100)$, since the total time of the interaction of the electron spin with the bath is only one oscillation period, i.e., roughly $1 /(A \sqrt{N})$, while the angular velocity is of order of $A / 4$. Furthermore, this error is easily recoverable by application of a correcting pulse of the external field $H_{z}$. Therefore, a physically meaningful measure of errors associated with decoherence of the $S^{x}$ operator is the total transverse component of the electron spin,

$$
s_{T}=\sqrt{s_{x}^{2}+s_{y}^{2}}
$$

which is proportional to $s_{x}^{(i)}$ if $s_{y}^{(i)}=0$. This is illustrated in Figs. 廿(a-d), where we plotted the oscillations of $s_{x}$ and $s_{T}$ for $\Delta P=0$ (left column, (a) and (c)), and for $\Delta P=0.2$ (right column,(b) and (d)), for the initial condition $s_{y}^{(i)}=s_{z}^{(i)}=0, s_{x}^{(i)}=1$. The solid lines correspond to $N=20$, and the dashed lines correspond to $N=10^{4}$. It is important that during the first oscillation
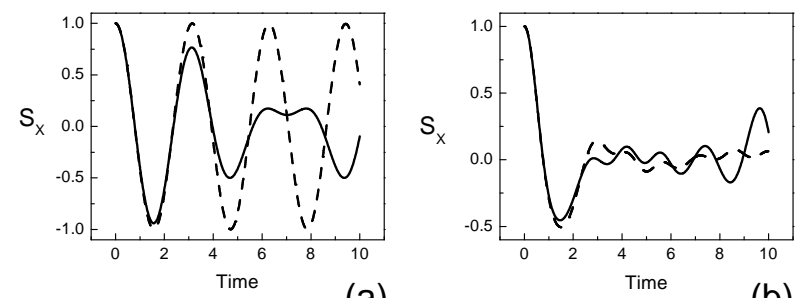

(a)
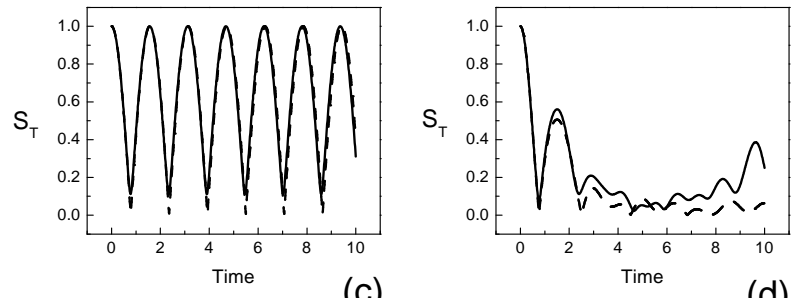

(b)

(c)
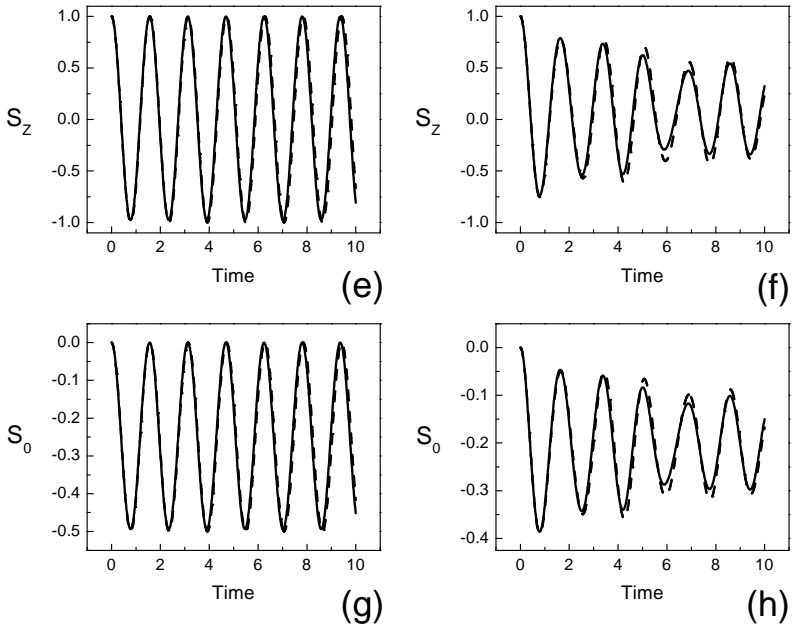

FIG. 1: First step of the protocol, encoding. Oscillations are shown for $s_{x}(\mathrm{a}, \mathrm{b}), s_{T}(\mathrm{c}, \mathrm{d}), s_{z}(\mathrm{e}, \mathrm{f})$ and $s_{0}(\mathrm{~g}, \mathrm{~h})$, obtained for $\Delta P=0(\mathrm{a}, \mathrm{c}, \mathrm{e}, \mathrm{g})$ and for $\Delta P=0.2(\mathrm{~b}, \mathrm{~d}, \mathrm{f}, \mathrm{h})$, with $N=20$ (solid curves) and $N=10^{4}$ (dashed curves).

period, the results for $s_{T}$ obtained with $N=20$ are close to those obtained with $N=10^{4}$, so that $N=20$ is sufficient to reliably estimate (with the precision of $\sim 0.05$ ) the fidelity of the quantum memory protocol at much larger values of $N . s_{T}$ is one of the three performance measures we use for the remainder of the paper.

In addition to the transverse errors, the oscillations of the value $s_{z}$ of $S^{z}$ are not simply proportional to the initial value $s_{z}^{(i)}$, so that the retrieved final value $s_{z}^{(f)}=$ $u_{1}+u_{2} s_{z}^{(i)}$ (i.e., the value $s_{z}^{(f)}$ is linearly dependent on $s_{z}^{(i)}$ but not proportional to it). This type of error is, as far as we know, irrecoverable. To quantify it, we consider the value of $s_{z}^{(f)}$ for the case $s_{z}^{(i)}=1, s_{x}^{(i)}=s_{y}^{(i)}=0$, and the value of $s_{z}^{(f)}$ for the initial conditions $s_{x}^{(i)}=1, s_{y}^{(i)}=$ $s_{z}^{(i)}=0$. For the latter case, the value $s_{z}^{(f)}$ is denoted as $s_{0}$, to distinguish it from the value $s_{z}^{(f)}$ obtained with the initial conditions $s_{z}^{(i)}=1, s_{x}^{(i)}=s_{y}^{(i)}=0 . s_{z}$ and $s_{0}$ are the remaining two performance measures used in 

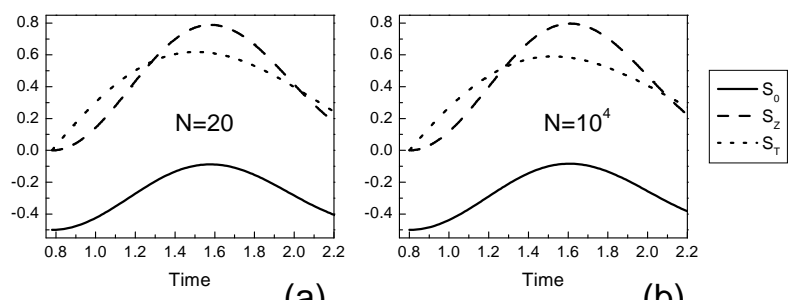

(a)

(b)

FIG. 2: Final step of the protocol, retrieval. The values $s_{0}$ (solid line), $s_{z}$ (dashed line) and $s_{T}$ (dotted line) are shown, obtained for $\Delta P=0.2$ with $N=20$ (a) and $N=10^{4}$ (b).

the paper. In ideal situation of $\Delta P=0, A_{k}=A$, the value $s_{0}$ should be zero, while in a non-ideal situation, $s_{0}$ quantifies the protocol error. Figs. [1 $(\mathrm{e}-\mathrm{h})$ illustrate this point, showing the oscillations of $s_{z}$ and $s_{0}$ for $\Delta P=$ 0 (left column, (e) and $(\mathrm{g})$ ), and for $\Delta P=0.2$ (right column,(f) and (h)). The solid lines correspond to $N=$ 20 , and the dashed lines correspond to $N=10^{4}$. Again, during the first oscillation period, the results obtained with $N=20$ are close to those obtained with $N=10^{4}$, so that $N=20$ is sufficient to reliably estimate the fidelity of the quantum memory protocol.

Now we study performance of the quantum memory protocol at a quantitative level. The goal is to ensure that consideration of $N=20$ is sufficient for making conclusions about the realistic case of $N \sim 10^{4}-10^{6}$. For $A_{k}=A$, using the Eqs. 23. the evolution of the reduced density matrix of the electron spin can be computed for the whole two-step protocol for $N \leq 10^{8}$. The spin ejection is equivalent to a measurement of $S^{z}$ described as a von Neumann's projection, so that at the ejection time $t=t_{e}$, the wave function

$$
\left|\psi\left(t_{e}\right)\right\rangle=|\uparrow\rangle \otimes\left|\nu_{1}\right\rangle+|\downarrow\rangle \otimes\left|\nu_{2}\right\rangle
$$

is transformed into a mixed state with the compound system's density matrix

$$
R_{e}=|\uparrow\rangle\left\langle\uparrow|\otimes| \nu_{1}\right\rangle\left\langle\nu_{1}|+| \downarrow\right\rangle\left\langle\downarrow|\otimes| \nu_{2}\right\rangle\left\langle\nu_{2}\right| .
$$

At the retrieval step, after injection of another electron in a dot, the compound system's density matrix becomes

$$
R_{r}=|\downarrow\rangle\langle\downarrow| \otimes\left(\left|\nu_{1}\right\rangle\left\langle\nu_{1}|+| \nu_{2}\right\rangle\left\langle\nu_{2}\right|\right) .
$$

The system's subsequent evolution can be again calculated using Eqs. 22 The resulting analytical expressions are complicated, but they can be easily estimated numerically for $N \leq 10^{8}$.

Fig. 2 illustrates the final step of the protocol, the retrieval of the initial state of the electronic spin, for $\Delta P=0.2$ for $\mathrm{N}=20$ (a) and $N=10^{4}$ (b). All three parameters quantifying the performance, $s_{T}, s_{z}$ and $s_{0}$, are shown for times close to the time of the state retrieval (half-period after the injection of the second electron), and one can see that the curves for $N=20$ almost exactly replicate the curves for $N=10^{4}$ (the difference is $\sim 0.05)$. Moreover, it is important to note that the maximum performance of the retrieval is achieved at slightly different times for $s_{T}, s_{z}$ and $s_{0}$. Below, we assume that the information retrieval is performed when $s_{z}$ reaches the maximum. In section IV we evaluate all three performance measures, $s_{z}, s_{T}$, and $s_{0}$, while calculation of the fidelity of the protocol for arbitrary quantum states is considered in section $\nabla$

\section{NUMERICS FOR $A_{k} \neq A$ : COMPUTATIONS FOR $N=20$ AND ANALYSIS}

In a real quantum dot, the electron density is nonuniform, so that the values of the hyperfine coupling constants $A_{k}$ differ substantially across the dot. Formally, the Hamiltonian (11) can be solved analytically for $A_{k} \neq A$ by Bethe ansatz ${ }^{21}$. However, this formal solution is impractical, especially in our problem, where the state of the bath has a complex form, with a large number of excited states, and where the non-unitary projection (see Eqs. 897 is an important part of the protocol. We are not aware of any way of employing the formally exact solution of Ref. 21 (which requires solving a system of order of $2^{N} / \sqrt{N}$ nonlinear equations) for practical quantitative estimates in such complex problems as the one considered here, even for a small number of the bath spins. For similar reasons, numerical approaches based on the P-representation of the spin density matrix ${ }^{22}$ are also not applicable. Instead, we use the numerical approach suggested in Ref. 16. 17: the direct solution of the time-dependent Schrödinger equation for the compound system (the electron spin plus the bath spins). This allows straightforward numerical modeling of the quantum memory protocol. The drawback of such an approach is that the exponential scaling of computation costs with $N$ limits simulations to $N \sim 20$ spins. Considering the above case of $A_{k}=A$, we have shown that $N=20$ is sufficient to make our simulations reflect larger $N$ behavior with a precision of order 0.05 .

In order to understand whether this remains true for $A_{k} \neq$ const, let us consider the case of perfect polarization $P=1$ which can be studied analytically with precision of $1 / N$ for large $N$. First, let us consider the electron initially in the state $\left|\phi_{0}\right\rangle=|\uparrow\rangle$ and the bath in the state $\left|\chi_{0}\right\rangle=|\downarrow, \downarrow, \ldots\rangle$. By acting with the Hamiltonian (11) on the state $\left|\psi_{0}\right\rangle \otimes\left|\chi_{0}\right\rangle$, we find that

$$
\mathcal{H}\left|\psi_{0}\right\rangle=\left(g_{e} \mu_{B} / 2\right)\left(H_{0}+H_{\mathrm{O} v h}\right)\left|\psi_{0}\right\rangle+(b / 2)\left|\psi_{1}\right\rangle
$$

where $H_{\mathrm{O} v h}=-\sum_{k} A_{k} /\left(2 g_{e} \mu_{B}\right)$ is the Overhauser field acting from the nuclei on the electron spin, $b=\sqrt{\sum_{k} A_{k}^{2}}$, and $\left|\psi_{1}\right\rangle=|\downarrow\rangle \otimes\left|\chi_{1}\right\rangle=|\downarrow\rangle \otimes\left[(1 / \sqrt{N}) \sum_{k} S_{k}^{+}\left|\chi_{0}\right\rangle\right]$. Analogous result for $\left|\psi_{1}\right\rangle$ can be presented in the form

$$
\begin{aligned}
\mathcal{H}\left|\psi_{1}\right\rangle & =-\left(g_{e} \mu_{B} / 2\right)\left(H_{0}+H_{\mathrm{O} v h}+M_{3} / M_{2}\right)\left|\psi_{1}\right\rangle(11) \\
& +(b / 2)\left|\psi_{0}\right\rangle+(b / 2)\left|u^{\prime}\right\rangle
\end{aligned}
$$

where $M_{n}=\sum_{k} A_{k}^{n}$ is the $n$-th moment of the distribution of the hyperfine couplings $A_{k}$ (correspondingly, 
$\left.b=\sqrt{M_{2}}\right)$, and the extra state $\left|u^{\prime}\right\rangle$ is orthogonal to both $\left|\psi_{0}\right\rangle$ and $\left|\psi_{1}\right\rangle$. By choosing the external field equal to its optimal value

$$
H_{0}=-H_{\mathrm{O} v h}-M_{3} /\left(2 M_{2} g_{e} \mu_{B}\right)
$$

and subtracting a constant from the Hamiltonian, we have

$$
\begin{aligned}
& \overline{\mathcal{H}}\left|\psi_{0}\right\rangle=(b / 2)\left|\psi_{1}\right\rangle \\
& \overline{\mathcal{H}}\left|\psi_{1}\right\rangle=(b / 2)\left|\psi_{0}\right\rangle+(b / 2)\left|u^{\prime}\right\rangle
\end{aligned}
$$

where $\overline{\mathcal{H}}=\mathcal{H}+M_{3} /\left(2 M_{2}\right)$.

This system is "practically" closed, since the norm of the extra state $\left|u^{\prime}\right\rangle$ is small for large $N,\left\|u^{\prime}\right\|^{2}=$ $\left[\left(M_{4} / M_{2}^{2}\right)-\left(M_{3}^{2} / M_{2}^{3}\right)\right] \sim 1 / N$ for a "non-pathological" distribution of $A_{k}$. As a result, the calculations for both the encoding and the retrieval steps can be done explicitly with the precision of $1 / N^{2}$. Introducing new basis states $\left|\psi_{+}\right\rangle=(1 / \sqrt{2})\left[\left|\psi_{1}\right\rangle+\left|\psi_{0}\right\rangle\right],\left|\psi_{-}\right\rangle=(1 / \sqrt{2})\left[\left|\psi_{1}\right\rangle-\right.$ $\left.\left|\psi_{0}\right\rangle\right]$, and $\left|u^{\prime} /\right|\left|u^{\prime}\right||\rangle$, the Hamiltonian $\overline{\mathcal{H}}$ can be represented by the matrix

$$
\overline{\mathcal{H}}=(b / 2)\left(\begin{array}{ccc}
1 & 0 & \delta \\
0 & -1 & -\delta \\
\delta & -\delta & 0
\end{array}\right)
$$

where $\delta=\left\|u^{\prime}\right\| / \sqrt{2}$. The unitary evolution during the encoding and the retrieval steps is described by the evolution operator $\exp (-i \overline{\mathcal{H}} t)$ which can be easily calculated, as well as the system's evolution during the projection step. We also note that the optimal times for encoding and for retrieval differ slightly (by the terms of order of $\left.\delta^{2}\right)$ from $\pi /(2 b)$, but this difference can be neglected since it leads to the corrections of order of $\delta^{3}$ during the encoding/retrieval steps.

By performing the straightforward calculations, one can arrive at the final answers for the performance parameters $s_{T}, s_{0}$, and $s_{z}$ :

$$
s_{0}=-8 \delta^{2}, \quad s_{T}=1-4 \delta^{2}, \quad s_{z}=1-16 \delta^{2}
$$

The deviation from ideal values is of order of $\delta^{2} \sim 1 / N$, which is about $5 \%$ for our simulations with $N=20$ (below, we will demonstrate the agreement in more detail).

In our simulations, two types of initial conditions are considered: (i) the electronic spin is in the state $s_{z}^{(i)}=1$, $s_{x}^{(i)}=s_{y}^{(i)}=0$, for evaluation of the $s_{z}$ performance measure, and (ii) the electronic spin is in the state $s_{x}^{(i)}=1$, $s_{z}^{(i)}=s_{y}^{(i)}=0$, for evaluation of the $s_{T}$ and $s_{0}$ performance measures. Then we can use the values $s_{z}, s_{T}$, and $s_{0}$ to determine the fidelity of the protocol (see section $\nabla$. In order to simulate the initial state of the polarized bath, we take the bath's state as a random superposition $|r\rangle$ of all possible basis states, apply the operator $\exp \left(-\gamma I^{z}\right)$ to the state $|r\rangle$ (such an operator is easily implemented using the Chebyshev's polynomials expansion described in detail in Ref. 16 17), and normalize the
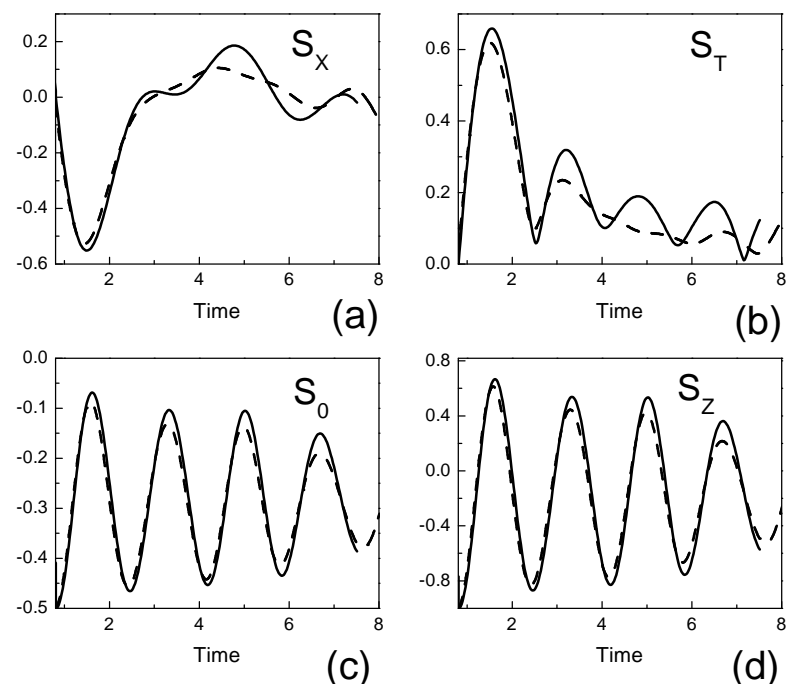

FIG. 3: Final step of the protocol, retrieval. The values $s_{x}(\mathrm{a}), s_{T}(\mathrm{~b}), s_{0}(\mathrm{c})$, and $s_{z}(\mathrm{~d})$ are shown, obtained for $\Delta P=0.2$ with $N=20$. The solid curves represent the results obtained with the bath's initial conditions obtained by application of the operator $\exp \left(-\gamma I^{z}\right)$ to the random state, the dashed curves correspond to the analytical solution.
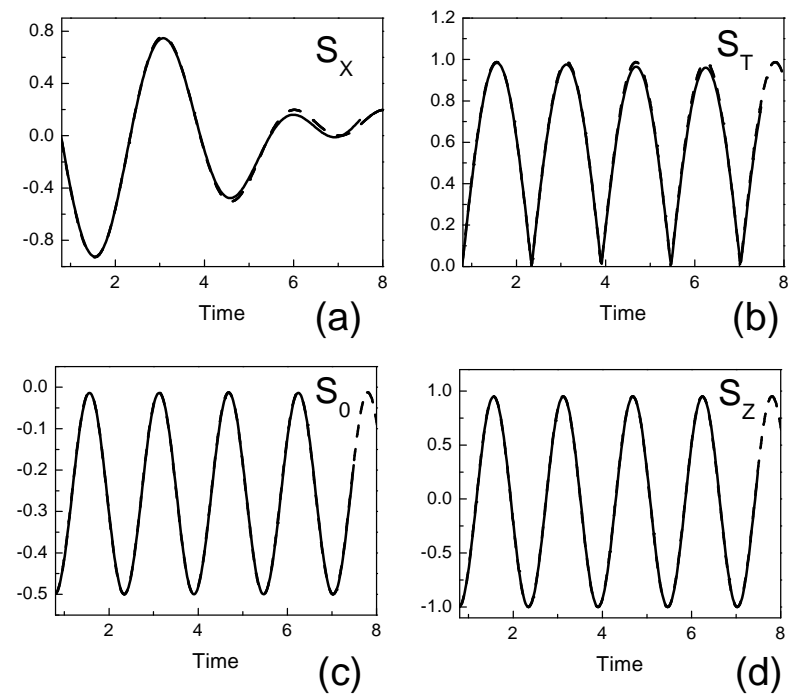

FIG. 4: Final step of the protocol, retrieval. The values $s_{x}$ (a), $s_{T}(\mathrm{~b}), s_{0}(\mathrm{c})$, and $s_{z}$ (d) are shown, obtained for $\Delta P=0$ with $N=20$. The solid curves represent the results obtained with the bath's initial conditions obtained by application of the operator $\exp \left(-\gamma I^{z}\right)$ to the random state, the dashed curves correspond to the analytical solution.

resulting state. Such a state corresponds to the bath's density matrix $\rho=(1 / Z) \exp \left(-\gamma I^{z}\right)$.

This construction of the bath's initial conditions can lead to statistical errors if the number of relevant states is not large enough. To ensure that the constructed initial condition is valid for $N=20$, we did the simulations with $A_{k}=A$, and compared them with the analytical 


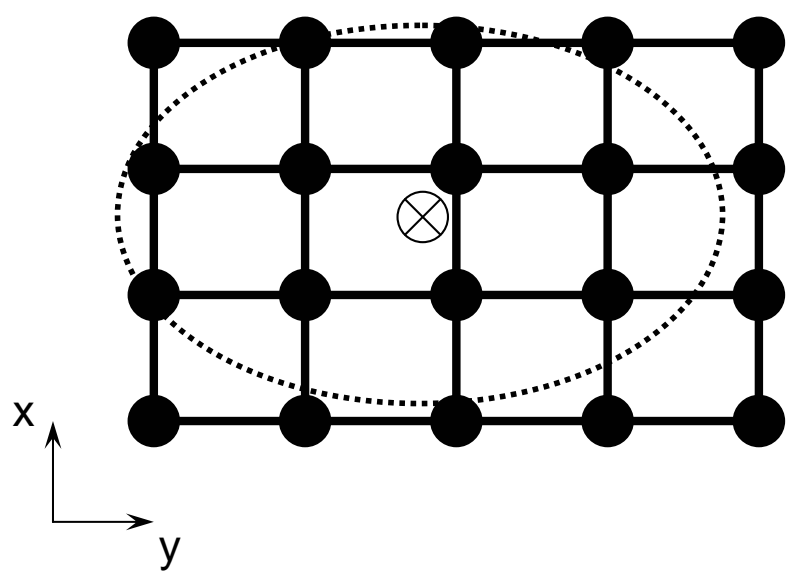

FIG. 5: The arrangement of the lattice of the bath spins and the electron density. The bath spins sites are marked by the solid circles, and the position of the maximum of the electron density is marked as $\otimes$; the dashed line schematically shows the contour line where the electron density is equal to $\exp (-1 / 2)$ of its maximum value.

results given in the previous section. The comparison is presented in Figs. 34 for $\Delta P=0.2$ (Fig. 3) and $\Delta P=0$ (Fig. (4), for $s_{z}, s_{0}, s_{T}$, and also for $s_{x}$ itself. Even in worst case of $\Delta P=0.2$, the simulation results remain close to the analytical ones, and at times close to the information retrieval time, the difference does not exceed $\sim 0.05$. For smaller $\Delta P$, the difference is smaller, and

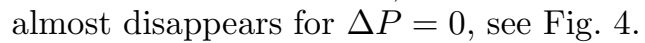

For the simulations, we consider $N=20$ bath spins which are placed at the sites of a $4 \times 5$ piece of a rectangular lattice with the lattice constants $a_{y}$ and $a_{x}$. We assume that the electrostatic potential inside the dot can be approximated as a 2-D harmonic well, so that the electron density in the dot is a 2-D Gaussian with the half-widths $w_{x}=(3 / 2) a_{x}$ and $w_{y}=2 a_{y}$ along the $x$ and $y$-axes. We set the maximum of the electron density is slightly displaced with respect to the center of the lattice by $l_{y}=0.2, l_{x}=0.1$ to prevent artificial symmetry effects. This arrangement is schematically shown on Fig. [5 where the bath spins' sites are marked by the solid circles, and the position of the maximum of the electron density is marked as $\otimes$; the dashed line schematically shows the contour line where the electron density is equal to $\exp (-1 / 2)$ of its maximum value. The values of $A_{k}$ were taken proportional to the electron density with the proportionality factor 1 , and were spread from 0.96 to 0.31 .

The external field $H_{0}$ in our simulations was taken equal to its optimal value, which compensates the Overhauser field $H_{\mathrm{O} v h}=-P \sum_{k} A_{k} /\left(2 g_{e} \mu_{B}\right)$ plus a small correction, $H_{0}=-H_{\mathrm{O} v h}-M_{3} /\left(2 M_{2} g_{e} \mu_{B}\right)$ (see Eq. 12). The memory protocol was simulated as follows:

1. The bath's initial polarized state was obtained by acting with the operator $\exp \left(-\gamma I^{z}\right)$ to the random state; the initial state of the electron spin was $|\uparrow\rangle$.
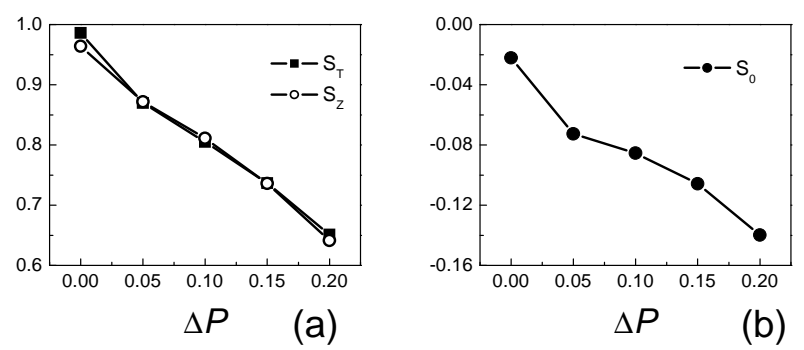

FIG. 6: Performance of the memory protocol as a function of $\Delta P$ for $w_{x}=(3 / 2) a_{x}$ and $w_{y}=2 a_{y}$. (a): the values of $s_{T}$ (solid squares) and $s_{z}$ (open circles); (b): the values of $s_{0}$ (solid circles). The values of $s_{T}$ and $s_{z}$ are very close to each other, and the corresponding curves almost coincide on the graph.
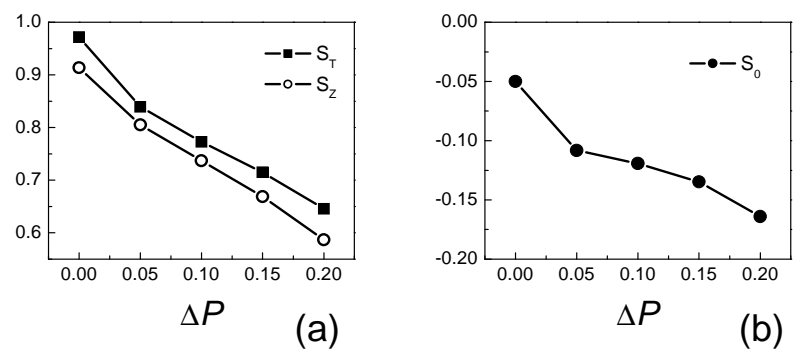

FIG. 7: Performance of the memory protocol as a function of $\Delta P$ for $w_{x}=3 a_{x} /(2 \sqrt{2})$ and $w_{y}=\sqrt{2} a_{y}$. (a): the values of $s_{T}$ (solid squares) and $s_{z}$ (open circles); (b): the values of $s_{0}$ (solid circles). In spite of noticeably larger spread of the couplings $A_{k}$, retrieval values decrease only by $5-7 \%$ (depending on $\Delta P)$ in comparison with Fig. [6]

2. The electron spin is allowed to interact with the bath, and the time when $s_{z}$ reaches its minimum is assumed to be the ejection time $t_{e}$.

3 . The ejection of the first electron is described according to Eq. 8

4. The second electron is injected in the state $|\downarrow\rangle$, so the compound system's density matrix has the form of Eq. 9

5. The second electron is allowed to interact with the bath, and the time when $s_{z}$ reaches its maximum is assumed to be the retrieval time $t_{r}$.

The fidelity of the protocol is indirectly determined by the values of $s_{T}, s_{z}$, and $s_{0}$ at time $t_{r}$ : for ideal memory, $s_{T}\left(t_{r}\right)$ and $s_{z}\left(t_{r}\right)$ would be one, and $s_{0}\left(t_{r}\right)$ would be zero.

The results for different values of $\Delta P$ are presented in Fig. 6] Both $s_{z}$ and $s_{T}$ are very close to each other, and both decrease approximately linearly with decreasing polarization. Similarly, $s_{0}$ deviates from zero linearly with decreasing polarization.

For comparison, in Fig. 7 we present the results for the case of $w_{x}$ and $w_{y}$ decreased by a factor of $1 / \sqrt{2}$ with respect to Fig. [6 (so that the dashed line of Fig. 5 corresponds now to the contour of the electron density equal 


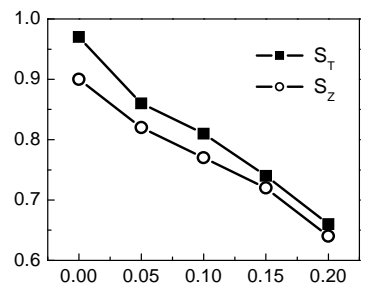

$\Delta P$

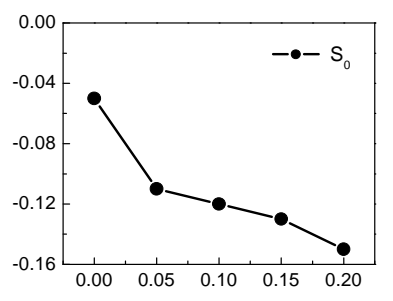

$\Delta P$
FIG. 8: Performance of the memory protocol as a function of $\Delta P$ for the unoptimized external field, for $w_{x}=(3 / 2) a_{x}$ and $w_{y}=2 a_{y}$. (a): the values of $s_{T}$ (solid squares) and $s_{z}$ (open circles); (b): the values of $s_{0}$ (solid circles). The maximum drop in performance in comparison with Fig. 6 is only about $6 \%$, and is noticeable only for small $\Delta P$.

to $\mathrm{e}^{-1}$ of the maximum value). This corresponds to a noticeably larger spread of $A_{k}$, from 0.92 to 0.09 . One can see that the perfromance of the protocol is determined by the value of $s_{z}$. In spite of considerably larger spread of $A_{k}$, the change in performance measures $s_{z}, s_{0}, s_{T}$ is small, from $5 \%-7 \%$ depending on the bath polarization. This is expected: the smaller values of $A_{k}$ give smaller contribution to the evolution, and, correspondingly, do not produce large errors in the course of the system's evolution.

We note here that the quantum memory protocol described in the introduction implies fine adjustment of the external field. The optimal value of $H_{0}$ includes the small correction $M_{3} /\left(2 M_{2}\right) \sim 1 / N$. While the Overhauser field can be measured rather precisely, the small correction $M_{3} /\left(2 M_{2}\right)$ could be hard to determine in practice. However, this is a $1 / N$ correction. According to our calculations, omission of this correction does not affect much the performance of the scheme. Fig. 8 shows the results obtained for the same parameters as Fig. [6] except for the unoptimized external field (i.e., $H_{0}=-H_{\mathrm{O} v h}$, without the correction $\left.M_{3} /\left(2 M_{2}\right)\right)$. The maximum drop in performance is only $6 \%$ (for $\Delta P=0$ ), and goes to almost zero with increasing $\Delta P$.

Thus, our results show that the quantum memory protocol remains feasible for realistic situations, where the polarization of the nuclear spins is incomplete, and the values of hyperfine couplings $A_{k}$ are spread over a large interval. Also, the external field does not need to be adjusted with precision of $1 / N \sim 10^{-4}$ for the quantum memory protocol to work. We believe that our results for $N=20$ provide adequate estimates of the performance of the protocol for realistic dots with $N=10^{4}$. In order to understand the corrections associated with extending from $N=20$ to larger $N$, let us compare the analytical estimates given in Eq. (15) with the numerical results. For the case shown in Fig. 6] the analytics gives $s_{T}=1.0-0.008, s_{0}=-0.016$, and $s_{z}=1.0-0.03$, while the numerical simulations give $s_{T}=1.0-0.014$, $s_{0}=-0.022$, and $s_{z}=1.0-0.036$. Similarly, for the case shown in Fig. [7] the analytics gives $s_{T}=1.0-0.021$, $s_{0}=-0.042$, and $s_{z}=1.0-0.084$, and the numerical results are $s_{T}=1.0-0.028, s_{0}=-0.05$, and $s_{z}=1.0-0.082$. The agreement between simulations and analytical results is reasonable, and we can consider our simulations to have a precision of $5-8 \%$.

\section{MINIMAL FIDELITY OF THE MEMORY PROTOCOL}

We now show how the values of $s_{0}, s_{T}$ and $s_{z}$ calculated above determine the fidelity of the memory protocol. The electron initially is in the state $\left|\phi_{0}\right\rangle=\alpha|\downarrow\rangle+\beta|\uparrow\rangle$, so that the electron's reduced density matrix initially is $\tau_{0}=\left|\phi_{0}\right\rangle\left\langle\phi_{0}\right|$, and the density matrix of the compound system is $R_{0}=\tau_{0} \otimes \rho$, where $\rho$ is the initial density matrix of the bath. At the end of the retrieval stage, the final state of the compound system is described by the density matrix $R_{f}$, so that the electron's reduced density matrix is $\tau_{f}=\operatorname{Tr}_{B} R_{f}$, where $\operatorname{Tr}_{B}$ is the trace over the bath spins. Ideally, we want $\tau_{f}=\tau_{0}$, but in reality the initial and the final density matrices of the electron are different. The fidelity for the protocol for the density matrix $\tau_{0}$ is $F_{\tau_{0}}=\operatorname{Tr}\left[\tau_{0} \tau_{f}\right]=\left\langle\phi_{0}\left|\tau_{f}\right| \phi_{0}\right\rangle$. Accordingly, the minimal performance of the memory protocol (the fidelity, $F$ ) is defined in a standard way ${ }^{23}$, as

$$
F=\operatorname{Min}_{\phi_{0}}\left[\left\langle\phi_{0}\left|\tau_{f}\right| \phi_{0}\right\rangle\right]
$$

which is minimized over all possible initial states $\left|\phi_{0}\right\rangle$.

To calculate the fidelity, we use the ideas of the quantum process tomography ${ }^{23}$. First, let us analyze in detail the dynamics of the density matrix. At the encoding stage, the evolution of the compound system is described by the unitary operator $U_{1}=\exp \left[-i \mathcal{H} t_{e}\right]$, so by the end of the encoding stage, the state of the compound system is $R_{1}=U_{1} R_{0} U_{1}^{\dagger}=U_{1}\left(\tau_{0} \otimes \rho\right) U_{1}^{\dagger}$. During the ejection of the first electron and injection of another one, the evolution is not unitary (see Eqs. 89); after injection of the second electron, the state of the compound system is:

$$
R_{e}=P_{\downarrow} R_{1} P_{\downarrow}+S^{-} R_{1} S^{+},
$$

where $P_{\downarrow}=|\downarrow\rangle\langle\downarrow|$ is the projection operator on the state $|\downarrow\rangle$ of the electron spin. At the retrieval stage, the evolution is again unitary, described by the operator $U_{2}=\exp \left[-i \mathcal{H}\left(t_{r}-t_{e}\right)\right]$ so the final state of the compound system is

$$
\begin{aligned}
R_{f} & =U_{2} P_{\downarrow} U_{1}\left(\tau_{0} \otimes \rho\right) U_{1}^{\dagger} P_{\downarrow} U_{2}^{\dagger} \\
& +U_{2} S^{-} U_{1}\left(\tau_{0} \otimes \rho\right) U_{1}^{\dagger} S^{+} U_{2}^{\dagger} .
\end{aligned}
$$

In order to analyze the evolution of the electron's reduced density matrix, we consider the evolution superoperator $\mathcal{L}$ for the electron spin

$$
\mathcal{L}\left[\tau_{0}\right]=\tau_{f}=\operatorname{Tr}_{B} R_{f}
$$

The superoperator $\mathcal{L}$ is linear. By representing the initial electron's density matrix in the form $\tau_{0}=(1 / 2) \mathbf{1}+b_{x} S^{x}+$ 
$b_{y} S^{y}+b_{z} S^{z}$, where $\mathbf{1}$ is the identity matrix $2 \times 2$, we obtain for the final density matrix $\tau_{f}$ :

$$
\tau_{f}=\mathcal{L}\left[\tau_{0}\right]=\tau_{f}^{0}+b_{x} \tau_{f}^{x}+b_{y} \tau_{f}^{y}+b_{z} \tau_{f}^{z}
$$

where

$$
\begin{aligned}
\tau_{f}^{0} & =\mathcal{L}[(1 / 2) \mathbf{1}], \quad \tau_{f}^{x}=\mathcal{L}\left[S^{x}\right], \\
\tau_{f}^{y} & =\mathcal{L}\left[S^{y}\right], \quad \tau_{f}^{z}=\mathcal{L}\left[S^{z}\right] .
\end{aligned}
$$

Each of the matrices $\tau_{f}^{0}, \ldots \tau_{f}^{z}$ can be expanded in the basis $1, S^{x}, S^{y}$, and $S^{z}$, so we just need to determine the expansion coefficients. This problem is considerably simplified by the fact that the superoperator $\mathcal{L}$ is invariant with respect to the rotations of the compound system in the $x-y$ plane, i.e. with respect to the operators $\exp \left[i a J^{z}\right]$, where $J^{z}=S^{z}+\sum_{k} I_{k}^{z}$ and $a$ is the rotation angle. For example, let us apply the rotation by the angle $\pi$ in the $x-y$ plane (the operator $\exp \left[i \pi J^{z}\right]$ ) to the matrix $\tau_{f}^{0}$. By doing that, we obtain that

$$
\operatorname{Tr}\left(\tau_{f}^{0} S^{x}\right)=-\operatorname{Tr}\left(\exp \left[-i \pi J^{z}\right] \tau_{f}^{0} \exp \left[i \pi J^{z}\right] S^{x}\right) .
$$

On the other hand, $\exp \left[-i \pi J^{z}\right] \tau_{f}^{0} \exp \left[i \pi J^{z}\right]=\tau_{f}^{0}$, so that $\operatorname{Tr}\left(\tau_{f}^{0} S^{x}\right)=0$. By applying similar symmetry arguments, we derive the following results:

$$
\begin{aligned}
\tau_{f}^{0} & =(1 / 2) \mathbf{1}+S^{z} u_{0} \\
\tau_{f}^{z} & =S^{z} w_{0} \\
\tau_{f}^{x} & =S^{x} v_{0} \cos \xi+S^{y} v_{0} \sin \xi \\
\tau_{f}^{y} & =S^{y} v_{0} \cos \xi-S^{x} v_{0} \sin \xi
\end{aligned}
$$

where the last equation is obtained by applying the operator $\exp \left[i(\pi / 2) J^{z}\right]$ (rotation by $\pi / 2$ in the $x-y$ plane) to the matrix $\tau_{f}^{x}$, and noticing that $\exp \left[-i(\pi / 2) J^{z}\right] S^{x} \exp \left[i(\pi / 2) J^{z}\right]=S^{y}$. In Eqs. 23. $u_{0}$, $w_{0}, v_{0}$, and $\xi$ are numerical parameters characterizing the fidelity, which are related in a simple manner to the parameters $s_{0}, s_{T}$, and $s_{z}$ obtained above from numerical simulations. We obtain $s_{z}$ by using the initial electron's density matrix $\tau_{0}=(1 / 2) 1+S^{z}$, so that $s_{z}=u_{0}+w_{0}$. Similarly, $s_{0}$ and $s_{T}$ are obtained from the initial electron's density matrix $\tau_{0}=(1 / 2) 1+S^{x}$, so that $s_{0}=u_{0}$ and $s_{T}=v_{0}$. We note that the action of the superoperator $\mathcal{L}$ transforming $S^{x}$ and $S^{y}$ into $\tau_{f}^{x}$ and $\tau_{f}^{y}$ involves the rotation in the $x-y$ plane by the angle $\xi$. As we explained above, this rotation can be, in principle, corrected, and we do not take it into account. Correspondingly, we neglect the reduction of fidelity caused by non-zero value of $\xi$, by putting $\xi=0$.

By combining Eqs. 20] and 23, the fidelity $F_{\tau_{0}}$ for a given initial density matrix $\tau_{0}=(1 / 2) \mathbf{1}+b_{x} S^{x}+b_{y} S^{y}+$ $b_{z} S^{z}$ can be expressed as

$$
F_{\tau_{0}}=(1 / 2)\left[1+v_{0}+b_{z} u_{0}+b_{z}^{2}\left(w_{0}-v_{0}\right)\right],
$$

where we used the fact that for initial pure state of the electron, $b_{x}^{2}+b_{y}^{2}=1-b_{z}^{2}$, and we need to minimize the fidelity (23) over all possible values of $b_{z}$, i.e. over all $b_{z} \in$ $[-1,1]$. The minimum value of this expression is achieved either at the extremum point $b_{z 0}=-u_{0} /\left[2\left(w_{0}-v_{0}\right)\right]$, or at the ends of the interval. Correspondingly, the minimal fidelity $F_{\mathrm{Min}}$ of the protocol is equal to the smallest of the three quantities

$$
\begin{aligned}
& F_{1}=\frac{1+s_{z}}{2}, \\
& F_{2}=\frac{1+s_{z}-2 s_{0}}{2}, \\
& F_{3}=\frac{1}{2}\left(1+s_{T}-s_{0}^{2} /\left[4\left(s_{z}-s_{0}-s_{T}\right)\right]\right) .
\end{aligned}
$$

With this fidelity measure, our results described above and depicted in Figs. 6] 7] and 8, can be presented as a single graph, Fig. 9] One can see that the minimum fidelity is $F_{\text {Min }} \sim 75 \%$ for $P=80 \%$, while as $P \rightarrow 100 \%$, $F_{\text {Min }} \rightarrow 96 \%$. The fact that the latter value is different from $100 \%$ is due to the modest number of bath spin $N$. Indeed, as seen from Eq. 15 for large $N$, the value of $F_{\text {Min }}$ for completely polarized bath differs from unity by the terms of order of $1 / N$, and therefore goes to zero for large $N$.

Using the analytical approach described in Sec. II we can estimate the minimum fidelity for small $\Delta P$ in the approximation of all $A_{k}=A=$ const. Indeed, the most significant contribution to $s_{z}, s_{0}$, and $s_{T}$ is given by the states with $I=-M$ (note that in our consideration $M<0)$; the contribution of the states with $I=-M+n$ is of order $(\Delta P)^{n}$ (where $n$ is a positive integer). Thus, to calculate the values $s_{z}, s_{0}$, and $s_{T}$ up to linear terms in $\Delta P$, we take into account only two states, $I=-M$ and $I=-M+1$, which have relative statistical weight $1-\Delta P / 2$ and $\Delta P / 2$, correspondingly, and perform averaging over all $M$ according to Eq. 3] The calculations give the following results:

$$
\begin{aligned}
& s_{z}=1-\frac{\Delta P}{2}\left(5+\cos ^{4} \gamma_{0}+\sin ^{4} \gamma_{0}\right)+O\left((\Delta P)^{3 / 2}\right) \\
& s_{0}=-\frac{\Delta P}{4}\left(5-\cos ^{4} \gamma_{0}-\sin ^{4} \gamma_{0}\right)+O\left((\Delta P)^{3 / 2}\right) \\
& s_{T}=1-\frac{\Delta P}{2}\left(2-\cos \gamma_{0}\right)+O\left((\Delta P)^{3 / 2}\right)
\end{aligned}
$$

where $\gamma_{0}=\pi / \sqrt{2}$, and from these values we obtain

$$
F_{\mathrm{Min}}=1-1.38386 \Delta P+O\left((\Delta P)^{3 / 2}\right) .
$$

This line is shown in Fig. 9] as a dashed line. The difference between this analytical result and the numerical data comes either from the assumption $A_{k}=A=$ const used in the derivation above, and from the $(\Delta P)^{3 / 2}$ corrections, which can be important for $\triangle P 0.1$.

Visible deviation of the fidelity from unity at $100 \%$ polarization results from finite size of the sample used in our simulations. Indeed, the analytical calculations of Ref. 11 and Section [V] of this paper suggests that at $100 \%$ polarization the error arises from the inhomogeneous hyperfine coupling, and hence scales as $1 / \sqrt{N}$. 


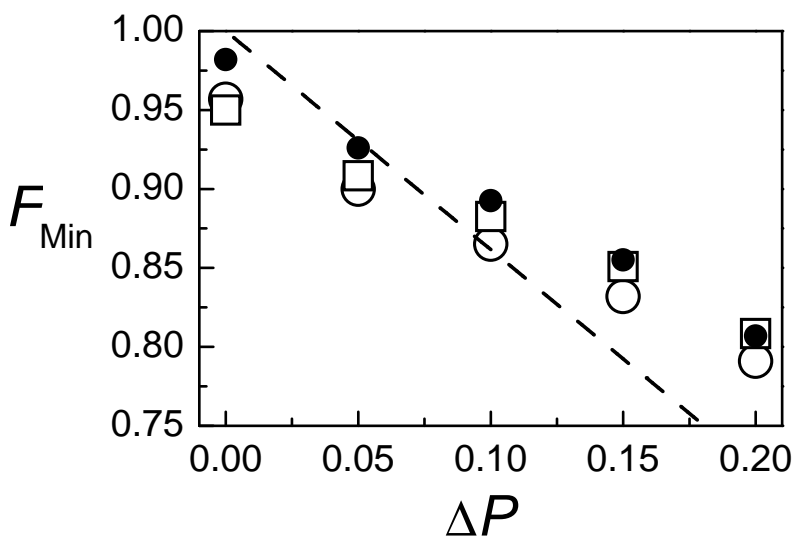

FIG. 9: Minimal fidelity $F_{\text {Min }}$ of the memory protocol as a function of $\Delta P$. Black circles: for $w_{x}=(3 / 2) a_{x}$ and $w_{y}=$ $2 a_{y}$ (same parameters as in Fig. 6). Open circles: for $w_{x}=$ $3 a_{x} /(2 \sqrt{2})$ and $w_{y}=\sqrt{2} a_{y}$ (same parameters as in Fig. 17). Open squares: unoptimized external field, for $w_{x}=(3 / 2) a_{x}$ and $w_{y}=2 a_{y}$ (same parameters as in Fig. 8). The dashed line corresponds to the analytical solution (26) for small $\Delta P$.

While it corresponds to few percent for the simulations presented in Fig. 9 $(N=20)$, for realistic $\operatorname{dots}\left(N=10^{6}\right)$, it is well below $10^{-3}$.

\section{CONCLUSIONS}

We study numerically the long-lived memory for electronic spin in a quantum dot taking into account the errors inherent in the protocol for a realistic system: the incomplete polarization of the bath of nuclear spins and the spread in the hyperfine interactions between the electron and the nuclei in the quantum dot. Although the imperfections deteriorate the fidelity of the quantum information retrieval, the scheme remains feasible for the spin bath polarizations exceeding $80 \%$, with a minimum fidelity of $F \sim 80 \%$. Fidelity is determined mostly by the retrieved value of $s_{z}$, and increases linearly with increasing polarization of the bath.

Since direct simulation of a real system with $\sim 10^{4} \mathrm{nu}-$ clear spins is currently impossible, we consider the system of $N=20$ spins coupled to the electron spin. Such a step requires justification, and accordingly a major part of our work is devoted to understanding the reliability of our simulations. By comparing different aspects of the numerical calculations with the analytical results, we show that our results give adequate estimates for realistic situations, with a precision of $5-8 \%$.

The errors considered in this work may be reduced by changes to the storage protocol or altered by considering of more realistic initial conditions. For example, it is expected that at high polarizations, created by electron spin-nuclear spin transfer techniques (e.g., Ref. 24), the nuclear spins may be in states with favorable symmetry properties 14 allowing for reduced error due to polarization. Similarly, estimating the current value of the nuclear spin polarization reduces the error associated with the uncertainty in polarization ${ }^{15,25}$. More detailed work will be necessary to consider the effects of these complexities.

\section{Acknowledgments}

V. V. D. thanks Harvard University for hospitality. This work was supported in part by the National Security Agency (NSA) and Advanced Research and Development Activity (ARDA) under Army Research Office (ARO) contracts DAAD 19-03-1-0132. This work was partially carried out at the Ames Laboratory, which is operated for the U. S. Department of Energy by Iowa State University under Contract No. W-7405-82 and was supported by the Director of the Office of Science, Office of Basic Energy Research of the U. S. Department of Energy.
1 D. Loss and D. DiVincenzo, Phys. Rev. A 57, 120 (1998).

2 J. M. Elzermanet al. Nature 430, 431 (2004).

3 A. C. Johnsonet al. Nature 435, 925 (2005).

4 J. Pettaet al. Science, 309, 2180 (2005).

5 A. Imamogluet al. Phys. Rev. Lett. 83, 4204 (1999).

6 A. S. Brackeret al., Phys. Rev. Lett. 94, 047402 (2005).

7 D. D. Awschalom, N. Samarth, and D. Loss, eds., Semiconductor Spintronics and Quantum Computation (SpringerVerlag, Berlin, 2002).

8 S. Datta and B. Das, Appl. Phys. Lett. 56, 665 (1990).

9 N. V. Prokof'ev and P. C. E. Stamp, Rep. Prog. Phys. 63, 669 (2000).

10 V. V. Dobrovitski, H. A. De Raedt, M. I. Katsnelson, and B. N. Harmon, Phys. Rev. Lett. 90, 210401 (2003).

11 J. M. Taylor, C. M. Marcus, and M. D. Lukin, Phys. Rev. Lett. 90, 206803 (2003).

12 J. M. Tayloret al. e-print: cond-mat/0407640 (2004).
13 C. Deng and X. Hu, e-print: cond-mat/0406478 (2004).

14 J. M. Taylor, A. Imamoglu, and M. D. Lukin, Phys. Rev. Lett. 91, 246802 (2003).

15 G. Giedke, J. M. Taylor, D. D'Alessandro, M. D. Lukin, and A. Imamoglu, e-print: quant-ph/0508144 (2005).

${ }^{16}$ H. De Raedt and V. V. Dobrovitski, "Decoherence in Quantum Spin Systems", in Computer Simulation Studies in Condensed-Matter Physics XVI, D. P. Landau, S. P. Lewis, and H.-B. Schüttler (eds.) (Springer Verlag, Berlin, Heidelberg, New York, 2004).

17 V. V. Dobrovitski and H. A. De Raedt, Phys. Rev. E 67, 056702 (2003).

18 D. Paget, G. Lampel, B. Sapoval, and V. Safarov, Phys. Rev. B 15, 5780 (1977).

19 J. Waugh, L. Huber, and U. Haeberlen, Phys. Rev. Lett. 20, 180 (1968).

20 L. M. Vandersypen and I. L. Chuang, Rev. Mod. Phys. 76, 
1037 (2004).

21 M. Gaudin, J. de Physique 37, 1087 (1976).

${ }^{22}$ Kh. Al-Hassanieh, V. V. Dobrovitski, E. Dagotto, and B. N. Harmon, cond-mat/0511681

23 M. A. Nielsen and I. L. Chuang, Quantum Computations and Quantum Information (Cambridge University Press,
Cambridge, 2002).

24 A. Imamoglu et al., Phys. Rev. Lett. 91, 017402 (2003).

25 D. Klauser, W. A. Coish, and D. Loss, e-print: cond-mat/0510177 (2005). 